El Antropoceno como proceso, no como estado José Luis Lanata, Nicolás Tanos, Adrián Monjeau

Relaciones, 46(2), e024, julio-diciembre 2021

ISSN 1852-1479 | https://doi.org/10.24215/18521479e024

https://revistas.unlp.edu.ar/relaciones

ISSN 0325-2221 (versión impresa)

Sociedad Argentina de Antropología (SAA)

Buenos Aires I Argentina

\title{
EL ANTROPOCENO COMO PROCESO, NO COMO ESTADO
}

\author{
José Luis Lanata*, Nicolás Tanos ${ }^{* *}$ y Adrián Monjeau****
}

Fecha de recepción: 28 de noviembre de 2020

Fecha de aceptación: 15 de junio de 2021

\section{RESUMEN}

La reciente propuesta de una nueva era geológica denominada Antropoceno a partir del año 1950, en donde el Homo sapiens es el principal actor, le ha dado a las ciencias humanas y sociales la posibilidad de discutir y contribuir en su adecuada definición, más allá del golden spike que se pueda emplear. Nuestro interés es mostrar que estamos frente a un proceso que arranca, quizás, hace 10.000-8.000 años y que los humanos, a través de nuestra capacidad de generar rápidamente nuevos nichos en diferentes dimensiones espacio temporales, hemos ido incrementado su aceleración con el paso del tiempo.

Palabras clave: Antropoceno - construcción de nicho - equilibrio puntuado - demografía - dinámica de cambio

THE ANTHROPOCENE AS A PROCESS, NOT AS A STATE

\section{ABSTRACT}

Recent proposal of the Anthropocene as a new geological era since 1950 AD, with Homo sapiens as its main actor, has presented the humanities and social sciences the opportunity of

\footnotetext{
* Consejo Nacional de Investigaciones Científicas y Técnicas, Instituto de Investigaciones en Diversidad Cultural y Procesos de Cambio, Universidad Nacional de Río Negro. E-mail: jllanata@conicet.gov.ar *** Universidad Nacional de Río Negro. E-mail: nicotanos@gmail.com

*** Consejo Nacional de Investigaciones Científicas y Técnicas. Fundación Bariloche. E-mail: amonjeau@ fundacionbariloche.org.ar
} 
contributing and discussing it in a relevant approach, beyond the golden spike that may be used. Our goal is to point out that we are facing a process that arose perhaps 10,000-8,000 years ago, in which humans, using our ability of swiftly generating new niches in different space-time dimensions, have accelerated it over time.

Keywords: Anthropocene - niche construction - punctuated equilibrium - demography change dynamic

\section{INTRODUCCIÓN}

Pensar que las demarcaciones de la huella humana son únicas, que ocurren en un momento particular, con impactos mundiales - a veces- y casi inmediatos, deja de lado la compresión de la multiplicidad de los procesos evolutivos tanto de nuestra especie como del total de aquellas que habitan nuestro planeta. Sería como si leyéramos el epílogo de un libro restándole importancia a sus capítulos; aquellos que nos dieron a conocer la trama esencial de la historia narrada y nos la hacen comprender adecuadamente. Sin duda, nuestra propia historia en el planeta es la de un proceso en continua transformación (Borrero 2011) y, mucho más, la de los últimos milenios. Es por eso que para comprender el Antropoceno (Crutzen y Stoermer 2000; Crutzen 2002) debemos ir más allá de sus efectos y consecuencias, las que en gran medida experimentamos actualmente. Es importante comprender y entender los múltiples procesos que lo generaron en una red de situaciones que se desarrollan tras la modificación y/o transformación de los nichos de los múltiples actores intervinientes y su impacto en las generaciones, ecosistemas y paisajes subsiguientes. Este conjunto de actores genera interacciones con esos cambios, los que, a su vez, fueron el motor de otros, generando una concatenación de situaciones y espacios que se modificaban con cada nueva generación (i.e. Steffen et al. 2007, Ellis et al. 2018).

En general, comprendemos antropocéntricamente las relaciones de nuestra especie con el resto del planeta. Si bien esto es lógico, nos lleva a olvidar y/o negar ciertos efectos directos y colaterales de los diferentes procesos que originan esas conductas. Es necesario reconocer sus distintas trayectorias; así, el conocimiento de nuestro pasado se transforma en la mejor herramienta analítica para cualquier tipo de acción por realizar en el presente y en el futuro. La puesta en escena del Antropoceno es una excelente oportunidad para ello (Lanata et al. 2017). El Antropoceno nos sitúa frente a una perspectiva donde los humanos somos el motor-factor-animal más relevante -quizás el mayor- en la aceleración de los cambios a nivel planetario (Crutzen y Stoermer 2000). Pero ¿somos algo más que un simple "actor geológico" que demarca el inicio de una nueva era, aproximadamente en 1950? Desde hace 10.000 años (Cochran y Harpending 2009; Erlandson y Braje 2013; Ellis et al. 2013) explotamos casi todos los ecosistemas planetarios generando nuevos comportamientos sociales de la mano de distintas innovaciones materiales que modifican nuestro nicho ecológico - así como el de otras especies-en diferentes bloques espacio-tiempo. Por lo tanto, comprender cómo se han ido construyendo esos nuevos nichos (sensu Odling-Smee y Laland 2012; Odling-Smee et al. 1996, 2003, etc.) nos lleva a romper con la dicotomía cultura-naturaleza (sensu Latour 2004, 2007, 2008) para entender cómo fue el dinamismo adaptativo de los humanos y sus distintos efectos -en los diferentes niveles, sociales, ambientales, corporales, etc.-.

Un número importante de científicos ha comenzado a debatir desde cuándo es posible que definamos el comienzo del Antropoceno (Ruddiman et al. 2015; Certini y Scalenghe 2015; Lewis y Maslin 2015; cfr. Briones et al. 2019; Svampa y Longa 2019). No obstante, otros puntos relevantes y poco discutidos son: a) desde cuándo esto es irreversible (Briones et al. 2019; Lanata et al. 2019) y, b) por qué los humanos hemos adquirido esta capacidad transformadora (Ellis 2016) en tal magnitud. Quizás, es porque hemos incrementado diferencialmente el proceso de construcción de nicho que todas las especies poseen (Smith y Zeder 2013; Cantrel et al. 2017; Ellis 
et al. 2018) y/o porque también sus diferencias regionales no tienen un correlato global en las distintas conductas humanas; es decir la humanidad no reacciona igual y uniformemente al mismo condicionamiento ambiental (Bauer y Ellis 2018), como lo tendrían los fenómenos ambientales en general (ver Gayo et al. 2019). ${ }^{1}$ Esto nos hace preguntar acerca de la humanidad y su impacto en el planeta a pesar de su efímero paso por el este -tan solo ca. 250.000 años (Lahr y Foley 1994, 1998; Stringer 2002, 2016; entre otros) vs. 4.000 millones de vida biológica sobre la superficie terrestre-. Durante los últimos milenios, los efectos del ser humano sobre los ambientes se han intensificado de tal manera que dieron lugar a honrarnos en tener nuestra propia era geológica. ${ }^{2}$ Sin duda, a medida que pasa el tiempo parece que los procesos se aceleran y se hacen más notables. Uno de ellos es el aumento demográfico. En los últimos tres siglos la población mundial se habría multiplicado por diez hasta llegar a los 7.8 mil millones de habitantes en 2020 y se estima 11 mil millones a fin del siglo XXI. ${ }^{3}$ Una de sus múltiples consecuencias es que explotamos aproximadamente el $50 \%$ de la superficie terrestre (Arias Maldonado 2016) para la producción de nuestros recursos. Uno de los varios puntos de inflexión del crecimiento demográfico de nuestra especie -por tomar, quizás, uno de los más cercanos- puede ubicarse de la mano del intercambio colombino (Crosby 1972; ver también Mann 2011; entre otros), vinculado con la incorporación global de nuevos recursos y fuentes de energía (Rapoport 1988). ${ }^{4}$ Es por ello que el aumento demográfico no es solo una característica de los últimos siglos, sino una resultante de múltiples factores a lo largo de milenios de cambio. Para entender esta "nueva realidad-situación" se hace necesario comprender el proceso que nos ha llevado al Antropoceno, que sin duda va más allá de la simpleza de señalar al Homo sapiens como motor de cambio a partir de año 1950 -o algunos años antes (ver detalles en Subramanian 2019, Baldwin y Erickson 2020, y también Biello 2015, Chomsky 2020, Syvitski et al. 2020)-.

\section{DISPERSIÓN GLOBAL Y DEMOGRAFÍA}

Teniendo en cuenta la juventud de los humanos (Lahr y Foley 1994, 1998; Stringer 2002, 2016; entre otros) vemos, en la dimensión de nuestro paso por el mundo, que es a partir de los últimos 10.000-8.000 años cuando comenzamos a dejar más huellas legibles a nivel planetario que muchas otras especies. Estas huellas no tienen una sola forma que la distingue, sino que son producto de diversas complejidades adaptativas que tienen particularidades espacio-temporales. Luego de $c a$. 190-160.000 años de permanencia solo en África y tras nuestras múltiples salidas fuera de ese continente (ver modelos en Lanata 2004), comienzan diferentes procesos de dispersión ${ }^{5}$ que culminan con la colonización de las masas continentales ca. 20-18.500 años atrás (Dillehay et al. 2015) en el sur de América. Tras la permanencia exclusivamente africana, nuestra dispersión global, lejos de ser homogénea, ha sido multifacética y pluridimensional, caracterizada por particularidades espacio-temporales variadas. Ellis et al. (2018; ver también Ellis 2015) señalan que, para comprender a largo plazo los cambios de nuestra especie, y su impacto en los ecosistemas, debemos considerar que las transformaciones de los nichos humanos son el resultado de una retroalimentación constante entre cambios culturales y ambientales, como lo muestra la múltiple evidencia arqueológica -ver artículos en Anthropocene 4, 2013-. De esta forma, sería posible comprender y abordar la problemática de la sostenibilidad a largo plazo a la que nos enfrenta el Antropoceno. Debemos comprender que se trata de un proceso de selección cultural de múltiples niveles (Wilson y Sober 1994). ${ }^{6}$ En el caso del Antropoceno, Ellis et al. (2018) consideran que un acercamiento de este tipo es el adecuado para comprender y determinar las relaciones causales, la forma y la intensidad de la construcción de nicho en la escala social humana. Estos autores señalan que para analizar las modificaciones del nicho humano hay que considerar: i) las escalas sociales relevantes, ii) el impacto de la herencia cultural, iii) cuáles son 
las innovaciones culturales latu sensu adoptadas, iv) la herencia ecológica, v) la forma en que se da la intensidad de construcción de nicho en un espacio-tiempo dado y, vi) los diferentes tipos de energía que se emplean -en términos de área per cápita, a mayor energía mayor ingeniería representada en los ecosistemas (ver Ellis et al. 2018:figura 1)-. Este acercamiento sociocultural de la construcción de nicho nos dice que cuando la densidad poblacional y el territorio de una sociedad se expanden, aumenta su impacto sobre los ambientes ya sea mediante sus conductas culturales como por las tecnologías y artefactos que emplea. Esto lleva a un mayor intercambio y/o cooperación, haciendo que la forma -ingeniería- en que se explotan los ecosistemas sea, en principio, supuestamente, más efectiva y compleja.

Cuando nos empezamos a agrupar en zonas específicas produciendo, importando y consumiendo recursos - cercanos y lejanos- comienza nuestra marca tangible en el medio ambiente. Los procesos de producción de recursos para la sostenibilidad de los grupos humanos que habitan estos espacios, por ejemplo, en núcleos urbanos quitan, transforman, desarman los ecosistemas cercanos en aras de alimentar estos conglomerados. Esta expansión multiespacial a paisajes desconocidos, en algunos casos con pocos o nulos recursos para la subsistencia, viene sucediendo desde cuando nos aventuramos fuera de África al ser una especia invasora en nuevos ecosistemas -ver diferentes mecanismos y resultados en Lockwood et al. (2007)-. Esta característica nos ha permitido explorar y explotar ecosistemas eficientemente -desde el punto de vista humano-. Pero desde hace unos 10.000-8.000 años nos encontramos con que nuestra especie habita en la mayor parte del planeta -salvo la Antártida y algunas islas del Océano Pacífico (Dewar y Wright 1993; Hunt y Lipo 2006; Elzinga 1993)-. No fueron un impedimento los espacios de difícil acceso, los climas extremos, etc., donde, a pesar de las locaciones desventajosas, la humanidad se las ha ingeniado para aprovechar gradualmente su flora y fauna con el fin de subsistir en primera instancia y, como efecto posterior, construir y desarrollar asentamientos urbanos, beneficiándose de recursos como el agua, los minerales y la energía (Cochran y Harpending 2009; Erlandson y Braje 2013) a través de innovaciones tecnológicas y sociales. Pensar en esto como procesos que hacen a la construcción de nicho y, consecuentemente, al Antropoceno, se condice con una serie de eventos que nos han llevado a un rápido cambio ambiental provocado, en una medida importante, por el impacto del aumento de la población humana y el incremento del consumo de recursos de diferente tipo. Por ejemplo, solo la intervención humana puede explicar por qué la concentración de dióxido de carbono y metano en la atmósfera presenta un aumento anómalo entre los siglos VI y III a.C., sustancialmente mayor que las fluctuaciones durante los miles de años previos (Petit et al. 1999, Trischler 2017).

En el plano ambiental, los humanos y las diferentes especies animales de las cuales obtenemos nuestra alimentación representan casi el $90 \%$ de toda la biomasa de mamíferos del planeta (Berger et al. 2020), y las actividades económicas relacionadas se han apropiado del 40\% de la producción primaria neta de la biósfera (Vitousek et al. 1986), necesitando utilizar más del 120\% de la biocapacidad planetaria para una adecuada sostenibilidad (Wackernagel et al. 2002). Esto ha sobrepasado los límites planetarios en varias dimensiones (Rockstrom et al. 2009). Su consecuencia es que, dadas las limitaciones de un sistema planetario finito, se produce el crecimiento de la biomasa humana en desmedro de la disminución de la biomasa de otras especies (Barnosky 2008). De hecho, como mencionamos, el fin de la dispersión de nuestra especie a nivel continental fue acompañada sincrónicamente con una serie de extinciones que se dieron durante el Pleistoceno final (Monjeau et al. 2017:figura 2) y que continua en el presente, superando ampliamente la tasa de extinción natural (Pimm et al. 1995). En un estudio reciente, Bar-On et al. (2018) señalan que, actualmente, la masa de todos los humanos combinada es más alta que la de todos los mamíferos terrestres juntos, y que esto tiene un impacto sin precedentes sobre la biomasa global no sólo de los mamíferos, sino también de peces, plantas y otros animales. Es por ello que este impacto humano sería el motor de una sexta gran extinción de especies (Kolbert 2014; Ceballos et al. 
2017) o que lo será en las próximas décadas de continuar estas tendencias (Barnosky et al. 2011). Somos una de las causas -quizás la más relevante- de un proceso que excepcionalmente ocurrió solo cinco veces en 600 millones de años, y que se debieron, las cinco veces anteriores, a cataclismos de escala planetaria (Maldonado 2009; Pimm et al. 2014). A los impactos mencionados se suma que, al finalizar el siglo XXI, podrían extinguirse de entre el 50\% y $90 \%$ de las seis mil novecientas lenguas endémicas actuales (Gorenflo et al. 2012). Muchas de las sociedades que hablan estas lenguas sobrevivían fuera de la lógica occidental hasta que los alcanzó, entre otros intrusos, el proceso de globalización de los últimos siglos (Wallerstein 1979). Existe una notable concurrencia espacial entre diversidad de lenguas y diversidad biológica debido a que la maquinaria de transformación de los paisajes acorraló a los pueblos originarios y a la biodiversidad en los mismos rincones adonde el mercado no ha llegado aún (Monjeau 2010).

Construir un nicho humano implica tomar materia y energía del stock natural que posee la biósfera (Odling-Smee et al. 2003) para convertirlo, a través de artefactos, recursos, costumbres, etc., en un medio para satisfacer las múltiples necesidades de un grupo en un espacio tiempo particular. La intensidad del cambio depende de la potencia disponible para lograr la transformación (Monjeau et al. 2015). En los últimos centenares de años, los logros tecnológicos han avanzado tanto que ya los humanos no reconocen la dependencia insoslayable que tenemos con la naturaleza (Descola 2005), ni tampoco diferencias entre lo natural y lo cultural (Latour 2004).

\section{CONSTRUYENDO NUEVOS NICHOS Y DEJANDO HUELLAS}

Reconocer los procesos que crean patrones permite entenderlos y modificarlos. La construcción de nicho conlleva una parte donde los comportamientos aprendidos y transmitidos socialmente modifican entornos. Los seres humanos, como otras especies, son constructores de nicho especialmente eficaces debido a su capacidad para generar cultura latu sensu (Smith 2007; Kendal et al. 2011; Arias Maldonado 2016), y más cuando son una especie invasora (Lanata et al. 2008). Los patrones de construcción de nicho tienen un sobre salto de tal implicancia biofísica que no hay posibilidad de vuelta atrás. Estas particularidades se diversifican con un mismo fin en los procesos de obtención de energía por parte de los humanos. Por más intensa que sea la modificación de un tipo de ecosistema a otro, el mundo "natural" nunca es reemplazado por completo, ya que siempre quedaran elementos del ecosistema anterior coexistiendo en el nuevo en un híbrido natural-cultural (Latour 2007). Somos la primera generación de científicos que podemos registrar estos procesos desde el espacio, a partir de las imágenes satelitales y poder ver la huella humana como una inmensa red interconectada (Sanderson et al. 2002). Al igual que en una red de pesca, la malla es un filtro que deja escapar a parte de la biota. Si es gruesa, fauna y flora pueden coexistir entre los grandes intersticios. En cambio, sí es fina, la fauna pequeña, alguna flora, hongos, virus y bacterias serán los únicos componentes que puedan coexistir con los humanos. No hay ninguna manera de esquivar la interacción -o unidad- humanos-naturaleza (Hall et al. 2001); los humanos impactamos en el mundo natural y el mundo natural impacta sobre nosotros (Laland et al. 1996, 2007; Ellis et al. 2018) modificando la calidad y cantidad de los servicios ecosistémicos que prodigan para el bienestar de la sociedad y generando anticuerpos que son las zoonosis (Quammen 2012). El problema de esta coexistencia es que hemos ocupado casi todos los ecosistemas, generando una biomasa de una sola especie de tal magnitud que es susceptible de propagar cualquier intercambio biótico por todo el planeta -tal como es el caso contemporáneo del COVID 19, la fiebre española de hace 100 años, solo por citar algunos-. Este proceso resulta relevante porque deviene en un ejemplo donde el aumento poblacional y la circunscripción social tienen un peso importante en el desarrollo de la intensificación. En el planeta se encuentran constantemente construcciones de nicho menores con sus propias particularidades. Esta réplica 
en diferentes lugares nos permite ver la red de construcción espacial como parte de una lógica adaptativa que se transforma en múltiples golden spike ${ }^{7}$ humanos.

\section{CONSTRUYENDO Y EXTINGUIENDO}

La huella humana es más profunda de lo esperado -ver desarrollo en Kitzes et al. 2008-. Esto queda registrado en su repercusión en las biotas que provoca una presión evolutiva/selectiva sobre las especies animales y vegetales con las que compartimos los ecosistemas. Esta presión estimula las respuestas coevolutivas y adaptativas, entre otras tantas. En algunos casos la reacción de las especies es favorable y se ajustan bien a una "convivencia"; en otros, puede resultar en la desaparición y/o extinción de uno de los dos ya sea a nivel local o general; o incluso puede sucederle lo mismo a un tercer actor como un colateral de la relación entre los primeros. Tomemos por ejemplo el caso de la dispersión de los humanos en las Américas. Hace ca. 30-25.000 años este continente estaba libre de humanos. Al comenzar nuestro proceso de dispersión se pudieron producir cambios/impactos en los diferentes ecosistemas con intensidades y repercusiones diversas. Y esto se debe sencillamente a que al iniciarse la dispersión no poseemos predadores naturales como fue el caso durante la permanencia exclusiva del Homo sapiens en África. Y a su vez, esto ocurre coetáneamente con los cambios característicos y propios de la transición Pleistoceno-Holoceno. La invasión americana del Homo sapiens tuvo lugar en un período relativamente corto de tiempo -en la escala de la especie (Lanata et al. 2008) - y coincidió con la extinción de un número importante de grandes vertebrados en muchos hábitats americanos (ver Grayson 1991; Meltzer 2015; Defler 2019; etc.). Si bien la causa última de tales extinciones todavía es controversial (ver Monjeau et al. 2017 y bibliografía allí citada), hay evidencias que apoyan tanto a los factores ambientales y biológicos como a los humanos. Lo más probable es que los tres factores y otros -nuevas bacterias y virus, ver por ejemplo Beltrame et al. (2020)- hayan desempeñado un rol relevante en una situación de estrés ecológico muy complejo. No nos adentraremos en este debate, pero nos interesa rescatar el tema de la competencia entre humanos y sus potenciales presas animales en una situación de dispersión/invasión inicial en un nuevo ecosistema.

Los resultados presentados sobre este particular por Monjeau et al. (2017) y Abramson et al. $(2017)^{8}$ muestran que en los momentos iniciales de la dispersión de los humanos, con estrategias de cazadores-recolectores, se desencadena la extinción del herbívoro que es competitivamente superior y la supervivencia del herbívoro que es competitivamente inferior (dentro del gremio de los herbívoros) pero que tiene mayor capacidad de dispersión, incluso en hábitats que permiten la convivencia con otros. Esto sucede aun cuando la presión de la depredación sea más baja sobre el herbívoro superior en relación con un herbívoro competitivamente inferior. En cuanto al rol que juegan el tamaño del sistema y las fluctuaciones poblacionales de los distintos actores, estos autores observan que la presencia de los humanos provoca un colapso ecológico cuando el sistema es de pequeño tamaño (por ejemplo, ocurre más rápido en islas que en continentes). El tiempo de extinción como función del tamaño del sistema, tal como calculan los autores en los modelos, muestra, además, la concordancia con los datos paleontológicos disponibles. Los resultados de Monjeau et al. (2017) y Abramson et al. (2017) rescatan la importancia de modelar el efecto combinado de los factores antrópicos y ambientales para describir apropiadamente su dinámica adaptativa en un ecosistema complejo donde se conjugan factores ecológicos humanos como preponderantes en el desarrollo de una biota cuando se generan interacciones no sustentables, como la aparición de un depredador en un nuevo espacio. Un punto interesante de estos modelos es que las presas no tienen la posibilidad de generar nuevos mecanismos de adaptación defensiva con relación al nuevo depredador. Esto hace que sufran desajustes poblacionales tales como la pérdida de tiempo generacional, que afecta la tasa neta de reproducción y, en consecuencia, la posibilidad de crecer 
demográfica y sosteniblemente en el lapso de una generación, lo que provoca la extinción. Por el contrario, las poblaciones humanas y sus presas menores tienden a tener el resultado opuesto.

El proceso resultante fue explicado por Rosenzweig y McCord (1991) como reemplazo del ocupante. Tras que los humanos ocupan estos ecosistemas con nuevas y, en un primer momento, eficaces estrategias, terminan reemplazando a alguna/s otra/s especie/s que no puede/n adaptarse a las nuevas situaciones. Se ha creado un nuevo nicho para una de las especies por lo que los humanos en este caso, poseen una serie de ventajas. Irán sustituyendo en la red trófica a otras especies; algunas desaparecerán, otras convivirán competitivamente o no. En el caso americano no se dio exactamente de esta manera entre humanos y la megafauna extinta. Los tiempos fueron relativamente cortos en términos evolutivos y, como se dijo antes, no dio lugar a ninguna acción para contrarrestarlo y hacerlo más prolongado, más teniendo en cuenta la transición ambiental en la que se desarrollaba. Se generó una carrera asimétrica donde las presiones selectivas fueron mucho más fuertes para las presas que para los cazadores. Los modelos citados nos dejan en claro que cualquiera hubiese sido la situación ambiental para la megafauna, su territorio y las características del ecosistema en el que se encontraban, su desaparición era casi inevitable. De esta manera, estos cazadores contribuyen activamente en la construcción y transmisión del nicho y con el transcurso del tiempo esta retroalimentación entre los individuos y el ambiente puede conducir a cambios evolutivos para la comunidad biológica generando problemas de rápida expansión en las poblaciones a través de la modificación de su medio lo que propicia procesos selectivos o de plasticidad fenotípica.

El americano es el último caso de colonización continental de nuestra especie con una estrategia cazadora-recolectora. No hemos comentado los otros -no es la finalidad aquí- pero sí es remarcable que han tenido tempos diferenciales en cada ecosistema continental, entre los que el de las América fue -quizás-el más rápido. Y cuando consideramos los seis puntos que nos alertan Ellis et al. (2018, ver supra) podemos comprender por qué los cazadores-recolectores se han mantenido durante tantos miles de años -más allá de pocas diferencias espacio-temporales- como un actor más, integrado dentro de los ecosistemas y subordinado a sus leyes y límites naturales. Así, los humanos tuvimos una biomasa importante, pero nunca una supremacía o predominancia que causara modificaciones de tal magnitud que produjera muchos cambios puntuados en sus nichos. A partir de esta situación, y con el impacto del paso del Pleistoceno al Holoceno, las modificaciones ecosistémicas globales, así como los cambios en las densidades demográficas -tanto humanas como de otros mamíferos- comenzamos a enfrentarnos a novedosos procesos de construcción de nicho. Esto hizo que en distintas partes del planeta surgieran innovaciones tecnológicas y sociales; embriones de una diversificación conductual, social y tecnológica del Homo sapiens que antes no se había experimentado (ver detalles en Braje y Erlandson 2013a). La extinción de especies, el aumento de animales domesticados y plantas cultivadas y la gran mezcla de especies entre los continentes (Crosby 1972) son algunos de los principales indicadores del Antropoceno, y no pueden revertirse aunque el cambio climático dé marcha atrás, ya que hay puntos de irreversibilidad instaurados (Lanata et al. 2019).

\section{INNOVANDO EN LA DIETA Y LA TECNOLOGÍA Y GENERANDO CIUDADES}

La evidencia de la ocupación humana en Monte Verde (Dillehay et al. 2015), extremo sur americano, nos dice que hace ca. 20-18.000 años atrás los humanos habían colonizado todas las masas continentales con estrategias cazadoras y/o recolectoras y/o pescadoras. Eran una especie más -única como todas $-{ }^{9}$ coexistiendo con otras en diferentes ecosistemas del planeta. Sin duda, la dinámica de la construcción del nicho humano considerada a nivel global no pudo ser igual y con el mismo tempo en cada ecosistema. Muy por el contrario, esta fue particular (ver Ellis et al. 
2018) en cada uno de los infinitos casos desde el cuerno de África hasta Patagonia, pero nunca con una magnitud tal que produjera una huella ecológica relevante (Braje y Erlandson 2013a) en el sentido que hoy la entendemos. Pero unos pocos milenios después -o quizás desde el Pleistoceno final (Braje y Erlandson 2013b; Trischler 2017)-, cuando ya estaban instaladas las condiciones ambientales propias del Holoceno, advertimos en diferentes ecosistemas terrestres los efectos de nuevas construcciones de nicho humanas. De la mano de; i) crecimiento poblacional, ii) cambios dietarios, iii) control y/o domesticación y translocación de determinadas especies vegetales y animales, iv) desarrollo de innovaciones tecnológicas y sociales, v) modificaciones en las estrategias de movilidad, y vi) limpieza de espacios, quema y deforestación de bosques -ya sean en conjuntos o no-, comenzamos a ver que nuestro impacto sobre los ambientes deja de ser mínimo y/o imperceptible en diferentes hot-spots del planeta.

La vinculación de un conjunto o de todos los puntos antes mencionados se dio de manera independiente en Cercano Oriente, China oriental, Nueva Guinea, Mesoamérica, Andes centrales, Norteamérica oriental y, posiblemente también, África subsahariana y Amazonia (Aikens y Lee 2013; McClure 2013, 2015; Roosevelt 2013; Boivin et al. 2016; Trischler 2017; etc.). Los humanos comenzamos a alterar grandes porciones del paisaje e intervinimos en el acervo genético de plantas y animales en una escala sin precedentes desde nuestro surgimiento como especie. De la mano de diferentes innovaciones -tecnológicas, sociales, etc.- (ver Harari 2011) se fueron dando nuevas formas de utilización de los recursos ecosistémicos. Para el caso del control y domesticación de animales, McClure (2015) hace hincapié en que, en la relación entre las especies animales elegidas, sus cambios y las modificaciones en los espacios donde se los mantenía, se ve claramente que primó el factor humano que generó cambios no solo a nivel demográfico, sino también en la incidencia en su pool genético, según las cualidades que más se necesitaran de esas especies. Pero, además, en esos espacios y en esas especies, y conforme al paso del tiempo, se observa que la energía necesaria originalmente se vuelve proporcionalmente pobre y/o ineficiente. Esto lleva a la necesidad de emplear más energía (Ellis et al. 2018) para lo que se desarrollan innovaciones tecnológicas y sociales que, con el tiempo, vuelven a necesitar más energía. La reducción de la movilidad como ahorro energético para, en principio, estar cerca de los nuevos recursos explotados contribuyó al desarrollo de los conglomerados sociales (Reyes-García et al. 2016), y con ellos los pueblos y ciudades, pero esto aumentó la huella ecológica. Y a la larga condujo a una ineficiencia energética.

Algunas de las consecuencias directas de la situación antes enunciada no solo nos remiten a las nuevas condiciones que resultan de la concentración poblacional en puntos específicos de los ecosistemas, sino también al crecimiento demográfico y la mayor explotación del paisaje en términos de los diferentes recursos y, por lo tanto, su impacto transformador. Si bien los casos pueden ser muchos luego de que nos dispersamos por todas las masas continentales, nos interesa destacar lo que Crosby $(1972,1986)$ denominó Intercambio Colombino e Imperialismo Ecológico -ver también Rapoport (1988)-. Crosby (1972) es uno de los primeros en alertarnos que para entender a los humanos no debemos separarlos de la naturaleza y, que para comprender su impacto global post siglo XVI -y tal vez antes-, debemos ver a nuestra especie como una con características invasivas. Este autor describe que, más allá de los relatos históricos relacionados con el impacto en los pueblos originarios, hay que analizar además la marca que dejamos en el resto de las especies animales y vegetales, así como en virus y bacterias. Y el intercambio generalizado de personas, plantas, animales, enfermedades e ideas entre los hemisferios oriental y occidental a partir del siglo XVI fue lo que, nuevamente, profundizó aún más la huella ecológica. Un punto interesante del intercambio colombino es que los nuevos invasores -esta vez con estrategias de colonos-se establecieron también en todos los ecosistemas templados (Crosby 1986), favoreciendo sobre todo el desarrollo de la biota portmanteau. ${ }^{10}$ Esto diezmó y transformó los ambientes en una magnitud antes no conocida, dando lugar a lo que el autor llamó imperialismo ecológico. 
Por otra parte, después de utilizar los recursos - tanto alimenticios como para la fabricación de artefactos, refugios, etc.- de los ecosistemas, devolvemos sus desechos al mismo ambiente $-\mathrm{u}$ otro-. Así modificamos, reorganizamos y/o transformamos los ecosistemas existentes o creamos nuevos para satisfacer nuestras múltiples necesidades.

Uno de los puntos centrales de este nuevo sistema -o imperialismo ecológico- está dado por el fortalecimiento de los conglomerados sociales. En gran medida los más destacados son los que llamamos urbanos, no solo por su magnitud, sino por la red de intercambio energético que éstos producen/necesitan. Su circunscripción espacial no se limita a los gradientes geográficos ya que explotan recursos que proceden de ambientes muy lejanos, incluso extracontinentales. En este sentido, las ciudades presentan un nuevo punto de irreversibilidad. Cuando una especie comienza a consumir más energía de la que produce transforma inevitablemente su ambiente -el circundante, así como los distantes- para las generaciones siguientes, quienes heredan un paisaje modificado/empobrecido, es decir, construyen un nuevo nicho. Monjeau et al. (2015) proponen para una cuantificación de la huella humana, una representación matemática para magnificar su impacto. Lo denominan factor épsilon $(\varepsilon)$ y nos permite ver el comportamiento de un recurso dado, permitiendo o inhibiendo la posibilidad de que tenga una dinámica sustentable. En otras palabras, nos dice que, a mayor huella humana, el factor $\varepsilon$ modifica las posibilidades futuras de construcción de nichos sustentables de las próximas generaciones. Estas cuestiones nos hacen insistir con la propuesta globalizadora en torno al Antropoceno. Recae en el proceso temporal como un reproductor de los procesos desmedidos de consumo, motor de progreso en el sistema capitalista, donde, en este caso, el factor $\varepsilon$ es un indicador matemático de acciones antiéticas, por ejemplo, el sobreuso de recursos, en el cual se ven afectadas varias partes y termina definiéndose básicamente en un escenario donde unos tienen y otros dejan de tener; espacio, recursos y posibilidades. También sirve para comprender que la huella humana es una cuestión inherente a la propia especie, la cual como venimos diciendo, en el momento que ingresa a un espacio o ecosistema, ya lo está transformando. La delimitación de algo semejante no solo puede estar atada a un solo evento o detonante, sino que tiene que poner énfasis en los procesos que los originaron.

Si tomamos la noción de Antropoceno como un proceso acumulativo de ciclos de construcción de nichos que vienen incrementando su velocidad que complejiza la unidad humanos-naturaleza, encontraremos que es vital comprender que, en pequeña escala, los espacios en los que se concentran grupos sociales vienen replicando este proceso por lo menos desde el Holoceno temprano. Esto señala el devenir de las ciudades y sus habitantes, que no es otra cosa que nuestro propio futuro antropocénico. Los procesos urbanos han intensificado las marcas antropogénicas produciendo tres características relacionadas entre sí: i) el desarrollo tecnológico acelerado, ii) el rápido incremento de la demografía humana, y iii) aumento del consumo de los recursos -y sus desperdicios-. ${ }^{11}$ El principal resultado ha sido la pérdida de paisajes bioclimáticos para dar paso a la actividad agropecuaria, minera, pesquera, petrolera, carreteras, ciudades y otras construcciones humanas -i.e. aviación, transporte marítimo-. También se ha reemplazado gran parte de la vida "natural" por animales y plantas domesticados para cubrir las crecientes necesidades alimentarias. Esto solo es visible, y se transforma en una necesidad, a partir de la aparición de grandes demandadores de recursos como son las ciudades. ${ }^{12}$

\section{CONSTRUCCIÓN DE NICHO, PROCESOS ANTROPOCÉNICOS Y LA MAGNIFICACIÓN DEL EQUILIBRIO PUNTUADO}

La mayoría de los sistemas manifiestan comportamientos que fluctúan entre distintos estados de equilibrio, sin que exista forzosamente un mecanismo de regreso a la "normalidad", lo cual ya sabemos que nunca hay una vuelta al mismo estado. Por el contrario, a largo plazo la reproduc- 
ción de estados aparentemente similares o vecinos termina por crear situaciones nuevas. Cuanto más cambian las condiciones, más abundan las oscilaciones entre estados distintos, provocando la dispersión de los elementos del sistema, que vagan en busca de nuevas configuraciones. Entonces, pensamos al nicho humano como un lugar de tensión-lucha permanente, explícita o no. La constante está determinada por una continua y cambiante utilización del ambiente, propia de una especie que tiene, entre otras características, la de la dispersión espacial. Los ecosistemas se caracterizan por su dinamismo, donde la comunidad biológica que lo habita está en constante interacción. El tema es saber el rol diferencial de cada actor de esa interacción y qué cambios importantes suceden cuando alguna especie de la comunidad cambia las formas de interacciones exponiéndolas más allá de los límites sostenibles de ese equilibrio circunstancial.

Desde que los humanos generamos registros de nuestras múltiples actividades, comenzamos a reconocer cómo influimos en el sistema terrestre, esto nos carga con la responsabilidad de ser también los primeros en querer en cambiar nuestro lugar en él. Nos hemos convertido en el principal agente de transformación del mundo, pero recién nos comenzamos a dar cuenta a principios del siglo XX -ver nota 2 -. Tenemos una intensidad, una velocidad, una capacidad de transformación como no se ha visto en otra especie (Ellis et al. 2013, 2018). Lo que sucintamente hemos descripto nos hace considerar que, teniendo en cuenta los principios del equilibrio puntuado -el cual explica que las especies se mantienen en un estado de estasis, con nulos o mínimos cambios durante largos períodos de tiempo, para sufrir en determinados momentos una «explosión evolutiva» durante la que se producen grandes cambios en cortos periodos de tiempo (Eldredge y Gould 1977)-, en los últimos 8-6 milenios, cada una de las mesetas que representan un aparente estado de estasis se acorta a medida que pasa el tiempo. Y que la explosión evolutiva que motivan los grandes cambios en cortos periodos, produce saltos cada vez más altos. ${ }^{13}$ Por lo tanto, no se produce un efecto lineal siempre, sino que parece dominar un efecto en mosaico o ramificado a nivel planetario. Estos efectos son los que caracterizarían al Antropoceno. Con la introducción del Antropoceno se pone en juego ese camino recorrido en conjunto que no hace otra cosa más que imbricar la relación; nada que toquemos sigue siendo algo natural, naturaleza es puramente lo que llamamos con ese nombre (Gamble 2002). Hay múltiples huellas, pero están unidas en un continuo, en un proceso constante, producto de las construcciones de nicho humanas. Ese proceso es el que ha producido y sigue produciendo lo que hemos llamado Antropoceno.

\section{AGRADECIMIENTOS}

Al FONCYT por el financiamiento de los PICT V 2014-1558 Sociedades y ambientes en transformación, y PICT IV-A 2019-02167 Sociedades y ambientes en transformación: De la TPH al Antropoceno y a todos sus integrantes. A los revisores por sus interesantes comentarios y sugerencias y a R.A.F. y M.M.L. por las múltiples charlas inspiradoras (JLL). Y no queremos dejar de hacerlo a nuestros estudiantes de Ecología y Evolución Humana-UNRN- por sus constantes e interesantes discusiones sobre el Antropoceno (JLL y NT) a lo largo de estos años.

\section{NOTAS}

1 Además, no debemos dejar de lado nuestra capacidad de transmitir información cultural de manera acumulada (Boyd y Richerson 1985) gracias a las diferentes formas que se iniciaron con la escritura circunscripta a determinadas élites en un principio; y a la cuasi-masificación digital contemporánea.

2 Si bien no es hasta comienzos del siglo XXI que comienza a hablarse del Antropoceno, la idea de que los humanos eran una importante fuerza de cambio fue expuesta primero por el científico ruso Vladimir 
Vernadsky en la década de 1920 cuando definió a la noosfera que, a diferencia de la biosfera, contenía una fuerte impronta de nuestra especie (ver Guillaume 2014).

3 Datos tomados de United Nations https://www.un.org/en/sections/issues-depth/population/index.html (acceso octubre 2020). Según Grombridge y Jenkins (2002), habríamos pasado de 1.000 millones en el siglo XVIII a 6.000 millones en el siglo XX.

4 Esto considera el impacto que tuvo a partir de los siglos XVI y XVII la incorporación de América y Oceanía al sistema europeo de la tríada ciencia-industria-capitalismo (sensu Harari 2017). Sin duda, el intercambio colombino aceleró los cambios y el crecimiento demográfico a escala planetaria, más allá de las propias oscilaciones en diferentes regiones.

5 Recordemos que la dispersión parecería ser una característica de nuestro género (Potts 1996).

6 La propuesta de Wilson y Sober (1994) hace hincapié en que también existen presiones selectivas a nivel de grupos de individuos las que, en muchos casos, son más fuertes que las que operan a nivel individual. Cuando esto sucede, los grupos sociales que actúan altruistamente poseen ventajas sobre lo que lo hacen egoístamente. Y estos comportamientos, instituciones, aprendizajes, innovaciones, etc., tienden a transmitirse más rápidamente. Esto fue analizado antropológicamente por Richerson y Boyd (2005), Henrich (2004) y Reyes-García et al. (2016).

7 El golden spike (clavo dorado) se utiliza de referencia visual a un límite cronoestratigráfico de un estratotipo concreto en relación a GSSP (Global Boundary Stratigraphic Sections and Points).

8 Estos trabajos modelan y discuten la extinción de megafauna en la transición Pleistoceno-Holoceno sobre la base de las principales dimensiones controversiales involucradas -ambiente, biota y actores-. La proyección matemática de extinción y coexistencia en un ecosistema genérico de predador-presa y considerando como presa dos herbívoros -superior e inferior en cuanto a tamaños-.

9 Utilizamos la idea de especie única siguiendo a Foley (1987).

10 Este es un término con el que define Crosby (1986) a la biota compuesta por plantas, animales, bacterias, gérmenes y virus que introdujeron los colonos europeos, y posteriormente otros,

11 Esta combinación ha dado lugar a un aumento del uso de minerales, combustibles fósiles, fertilizadores agrarios, extinción de especies y/o nuevas formas de domesticaciones, la modificación en los ecosistemas tanto terrestres como costeros.

12 UNESCO informa que la población mundial que vive en ciudades se ha triplicado desde 1950 y calcula que dos de cada tres humanos vivirán en ciudades en 2050. https://es.unesco.org/unesco-for-sustainable-cities.

13 Con esto no queremos decir que los procesos anagenéticos no tengan un rol importante en otras escalas espacios temporales.

\section{REFERENCIAS BIBLIOGRÁFICAS}

Abramson, G., M. F. Laguna, M. N. Kuperman, A. Monjeau y J. L. Lanata (2017). On the roles of hunting and habitat size on the extinction of Megafauna. Quaternary International 431: 205-215.

Aikens, C. M. y G. A. Lee (2013). Postglacial inception and growth of anthropogenic landscapes in China, Korea, Japan, and the Russian Far East. Anthropocene 4: 46-56.

Arias Maldonado, M. (2016). El giro antropocénico. Sociedad y medio ambiente en la era global. Política y Sociedad 53(3): 795-814.

Baldwin, A. y B. Erickson (2020). Introduction: Whiteness, coloniality and the Anthropocene. Environment and Planning D: Society and Space 38(1): 3-11.

Barnosky, A. D. (2008). Megafauna biomass tradeoff as a driver of Quaternary and future extinctions, PNAS 105(S1): 11543-11548. https://doi.org/10.1073/pnas.0801918105

Barnosky, A. D., N. Matzke, S. Tomiya, G. O. U. Wogan, B. Swartz, T. Quental, C. Marshall, J. McGuire, E. Lindsey, K. C. Maguire, B. Mersey y E. Ferrer (2011). Has the Earth's mass extinction already arrived? Nature 471: 51-57. 
Relaciones de la Sociedad Argentina de Antropología 46 (2), julio-diciembre 2021: 681-697

Bar-On, Y. M., R. Phillips y R. Milo (2018). The biomass distribution on Earth. PNAS 115: 6506-6511. doi/10.1073/pnas.1711842115

Bauer, A. M. y E. C. Ellis (2018). The Anthropocene divide. Obscuring understanding of social-environmental change. Current Anthropology 59: 209-227.

Beltrame, M. O., C. Pruzzo, R. Sanabria, A. E. Pérez y M. S. Mora (2020). First report of pre-Hispanic Fasciola hepatica from South America revealed by ancient DNA. Parasitology 147(3): 371-375. doi. org/10.1017/S0031182019001719

Berger J., T. Wangchuk, C. Briceno, A. Vila y J. E. Lambert (2020). Disassembled food webs and messy projections: Modern ungulate communities in the face of unabating human population growth. Frontiers in Ecology and Evolution 8 (128): DOI: 10.3389/fevo.2020.00128

Biello, D. (2015). Did the Anthropocene begin in 1950 or 50,000 years ago? Scientific American, April 2. https://www.scientificamerican.com/article/did-the-anthropocene-begin-in-1950-or-50-000-years-ago/

Boivin, N. L., M. A. Zeder, D. Q. Fuller, A. Crowther, G. Larson, J. M. Erlandson, T. Denham y M. D. Petraglia (2016). Ecological consequences of human niche construction: Examining long-term anthropogenic shaping of global species distributions PNAS 113(23): 6388-6396. doi.org/10.1073/pnas.1525200113

Borrero, L. A. (2011). The archaeology of transformation. Quaternary International 245 (1): 178-181.

Boyd, R. y P. J. Richerson (1985). Culture and the evolutionary process. Chicago. University of Chicago Press.

Braje, T. J. y J. M. Erlandson (2013a). Human acceleration of animal and plant extinctions: A Late Pleistocene, Holocene, and Anthropocene continuum. Anthropocene 4: 14-23.

Braje, T. J. y J. M. Erlandson (2013b). Archeology and the Anthropocene. Anthropocene 4: 1-7.

Briones, C., J. L. Lanata y A. Monjeau (2019). El futuro del Antropoceno. Utopía y Praxis Latinoamericana 84: 19-31.

Cantrell, B., L. J. Martin y E. C. Ellis (2017). Designing autonomy: Opportunities for new wildness in the Anthropocene. Trends in Ecology and Evolution 32(3):156-166. DOI: 10.1016/j.tree.2016.12.004

Ceballos, G., P. Ehrlich y R. Dirzo (2017). Biological annihilation via the ongoing sixth mass extinction signals by vertebrate population. PNAS 114(30) E6089-E6096. https://doi.org/10.1073/pnas.1704949114

Certini, G. y R. Scalenghe (2015). Holocene as Anthropocene. Science 349(6245): 246. doi: 10.1126/ science.349.6245.246-a

Chomsky, N. (2020). Cooperación o extinción. Barcelona. Penguin Random House y Ediciones B.

Cochran, G. y H. Harpending (2009). The 10,000 year explosion: How civilization accelerated human evolution. Nueva York, Basic Books.

Crosby, A. (1972). The columbian exchange: Biological and cultural consequences of 1492. Westport, Greenwood Pub. Group.

Crosby, A. (1986). Ecological imperialism: The biological expansion of Europe, 900-1900. Cambridge, Cambridge University Press.

Crutzen, P. J. (2002). Geology of mankind. Nature 415: 23. https://doi.org/10.1038/415023a 
Crutzen, P. J. y E. F. Stoermer (2000). The “Anthropocene”. Global Change Newsletter 41: 17-18.

Defler, T. (2019). History of terrestrial mammals in South America. Switzerland, Springer Natures.

Descola, P. (2005). Más allá de la naturaleza y la cultura. Etnografías Contemporáneas 1: 93-114.

Dewar, R. E. y H. T. Wright (1993). The culture history of Madagascar. Journal of World Prehistory 7: 417466. https://doi.org/10.1007/BF00997802

Dillehay, T. D., C. Ocampo, J. Saavedra, A. Oliveira Sawakuchi, R. M. Vega, M. Pino, M. B. Collins, L. Scott Cummings, I. Arregui, X. S. Villagran, G. A. Hartmann, M. Mella, A. González y G. Dix (2015). New archaeological evidence for an early human presence at Monte Verde, Chile. PLoS ONE 10 (11): e141923. DOI: 10.1371/journal.pone.0141923

Eldredge, N. y S. J. Gould (1977). Punctuated equilibria: The tempo and mode of evolution reconsidered. Paleobiology, 3(2): 115-151.

Ellis, E. C. (2015). Ecology in an anthropogenic biosphere. Ecological Monographs 85: 287-331.

Ellis, E. C. (2016). Why is human niche construction transforming Planet Earth? RCC Perspectives 5: 63-70.

Ellis, E. C., D. Q. Fuller, J. O. Kaplan y W. G. Lutters (2013). Dating the Anthropocene: Towards an empirical global history of human transformation of the terrestrial biosphere. Elementa: Science of the Anthropocene 1:000018 DOI:10.12952/journal.elementa.000018

Ellis, E. C., N. R. Magliocca, C. J. Stevens y D. Q. Fuller (2018). Evolving the Anthropocene: Linking multi-level selection with long-term social-ecological change. Sustainability Science 13: 119-128. DOI: 10.1007/s11625-017-0513-6

Elzinga, A. (1993). Antarctica: The construction of a continent by and for science. En: E. Crawford, T. Shinn y S. Sörlin (eds.), Denationalizing science: The contexts of international scientific practice: 73-106. Dordrecht, Kluwer.

Erlandson, J. M. y T. J. Braje (2013). Archeology and the Anthropocene. Anthropocene 4: 1-7.

Foley, R. A. (1987). Another unique species: Patterns in human evolutionary ecology. Michigan, Longman Science and Technology.

Gamble, C. (2002). Arqueología Básica. Barcelona, Ariel Prehistoria.

Gayo, E. M., V. B. McRostie, R. Campbell, C. Flores, A. Maldonado, M. Uribe-Rodríguez, P. I. Moreno, C. M. Santoro, D. A. Christie, A. A. Muñoz y L. Gallardo (2019). Geohistorical records of the Anthropocene in Chile. Elementa Science of the Anthropocene 7: 15. https://doi.org/10.1525/elementa.353

Gorenflo, L. J., S. Romaine, R. A. Mittermeier y K. Walker-Painemilla (2012). Co-occurrence of linguistic and biological diversity in biodiversity hotspots and high biodiversity wilderness areas. PNAS 109 (21): 8032-8037. https://doi.org/10.1073/pnas.1117511109

Grayson, D. K. (1991). Late Pleistocene mammalian extinctions in North America: Taxonomy, chronology, and explanations. Journal of World Prehistory 5: 193-231.

Groombridge, B. y M. Jenkins (2002). World atlas of biodiversity. Berkeley, University of California Press. Guillaume, B. (2014). Vernadsky's philosophical legacy: A perspective from the Anthropocene. The Anthropocene Review 1: 137-146. 
Relaciones de la Sociedad Argentina de Antropología 46 (2), julio-diciembre 2021: 681-697

Hall, C., D. Lindernberger, R. Kümmel, T. Kroeger y W. Eichhorn (2001). The need to reintegrate natural sciences with economics. Bioscience 51: 663-673.

Harari, Y. N. (2011). Veinte lecciones para el siglo XXI. Barcelona. Debate.

Harari, Y. N. (2017). Sapiens. De animales a dioses. Una breve historia de la humanidad. Barcelona. Debate.

Henrich, J. (2004). Cultural group selection, coevolutionary processes and large-scale cooperation. Journal of Economic Behavior \& Organization 53: 3-35. https://doi.org/10.1016/S0167-2681(03)00094-5

Hunt, T. L. y C. P. Lipo (2006). Late colonization of Easter Island. Science 311 (5767): 1603-1606. DOI: 10.1126/science. 1121879 .

Kendal, J. R., J. Tehrani y J. Odling-Smee (2011). Human niche-construction in interdisciplinary focus. Philosophical Transactions of the Royal Society B 366: 785-792.

Kitzes, J., M. Wackernagel, J. Loh, A. Peller, S. Goldfinger, D. Cheng, D. y K. Tea (2008). Shrink and share: humanity's present and future Ecological Footprint. Philosophical transactions of the Royal Society of London. Series B 363(1491): 467-475. https://doi.org/10.1098/rstb.2007.2164

Kolbert, E. (2014). The sixth extinction. An unnatural history. New York, Henry Holt and Co.

Lahr, M. M. y R. A. Foley (1994). Multiple dispersals and modern human origins. Evolutionary Anthropology 3: 48-60.

Lahr, M. M. y R. A. Foley (1998). Towards a theory of modern human origins: geography, demography, and diversity in recent human evolution. Yearbook of Physical Anthropology 41: 137-7.

Laland, K. N., J. R. Kendal y G. R. Brown (2007). The niche construction perspective: Implication for evolution and human Behavior, Journal of Evolutionary Psychology 5: 51-66.

Laland, K. N., F. Odling-Smee y M. W. Feldman (1996). On the evolutionary consequences of niche construction, Journal of Evolutionary Biology, 9, 293-316.

Lanata, J. L. (2004). Modelar el origen de los humanos modernos. Explorando algunos temas de Arqueología, En: A. M. Aguerre y J. L. Lanata (eds.), Explorando algunos temas de arqueología: 153-175. Barcelona, Gedisa.

Lanata, J. L., C. Briones y A. Monjeau (2017). La controversia sobre el Antropoceno como una oportunidad: una cuestión de enfoques en lugar de designaciones formales. Interciencia 42(3): 186-189.

Lanata, J. L., L. Martino, A. Osella y A. Garcia Herbst (2008). Demographic conditions necessary to colonize new spaces: the case for early human dispersal in the Americas. World Archaeology. 40: 520-537.

Lanata, J. L., A. Monjeau, C. Briones, M. N. Kuperman, G. Abramson y M. F. Laguna (2019). El camino irreversible del Antropoceno, Revista de la Escuela de Antropología XXV. https://doi.org/10.35305/ revistadeantropologia.v0iXXV.97

Latour, B. (2004). Politics of nature: How to bring the sciences into democracy. Harvard, University of Harvard.

Latour, B. (2007). Nunca fuimos modernos. Buenos Aires, Siglo Veintiuno.

Latour, B. (2008). Reensamblar lo social. Una introducción a la teoría del actor red. Buenos Aires, Manantial. 
Lewis, S. L. y M. A. Maslin (2015). Geological evidence for the Anthropocene. Science 349(6245): 246-247. DOI: $10.1126 /$ science.349.6245.246-b

Lockwood, J. L., M. F. Hoopes y M. P. Marchetti (2007). Invasion ecology. Oxford y Victoria, Blackwell Pub. Maldonado, C. E. (2009). Evolución, teoría de las extinciones, complejidad. Acta de Biología Colombiana 14: 283-300.

Mann, C. (2011). 1493: Uncovering the New World Columbus created. New York, Knopf Doubleday Publishing Group.

McClure, S. B. (2013). Domesticated animals and biodiversity: Early agriculture at the gates of Europe and long-term ecological consequences. Anthropocene 4: 57-68.

McClure, S. B. (2015). The pastoral effect, Niche construction, domestic animals, and the spread of farming in Europe. Current Anthropology 56: 901-910.

Meltzer, D. (2015). Pleistocene overkill and North American mammalian extinctions. Annual Review of Anthropology 44: 33-53.

Monjeau, A. (2010). El caso Ayui y la movediza frontera entre lo bueno y lo malo. La Ley, Agosto.

Monjeau, A., B. Araujo, G. Abramson, M. Kuperman, M. F. Laguna y J. L. Lanata (2017). The controversy space on Quaternary megafaunal extinctions. Quaternary International 431:194-204. dx.doi.org/10.1016/j. quaint.2015.10.022 ISSN 1040-6182

Monjeau, A., J. L. Lanata, G. Abramson, M. Kuperman y M. Laguna (2015). ¿Sustentabilidad? RedBioética UNESCO 6(11): 4-19.

Odling-Smee, F, J. y K. N. Laland (2012). Ecological inheritance and cultural inheritance: What are they and how do they differ? Biological Theory 6: 220-230.

Odling-Smee, F. J., K. N. Laland y M. W. Feldman (1996). Niche construction, American Naturalist 147: 641-648.

Odling-Smee, F. J., K. N. Laland y M. W. Feldman (2003). Niche construction: The neglected process in evolution, Princeton, Princeton University Press.

Petit, J. R., J. Jouzel, D. Raynaud, N. I. Barkov, J. M. Barnola, I. Basile, M. Bender, J. Chappellaz, M. Davis, G. Delaygue, M. Delmotte, V. M. Kotlyakov, M. Legrand, V. Y. Lipenkov, C. Lorius, L. Pépin, C. Ritz, E. Saltzman y M. Stievenard (1999). Climate and atmospheric history of the past 420,000 years from the Vostok ice core, Antarctica. Nature 399:429-436. doi.org/10.1038/20859

Pimm, S. L., C. N. Jenkins, R. Abell, T. M. Brooks, J. L. Gittleman, L. N. Joppa, P. H. Raven, C. M. Roberts y J. O. Sexton (2014). The biodiversity of species and their rates of extinction, distribution, and protection. Science 344: 6187 1246752. DOI: 10.1126/science. 1246752

Pimm, S. L., G. J. Russell, J. L. Gittleman y T. M. Brooks (1995). The future of biodiversity. Science 269: 347-350.

Potts, R. (1996). Humanity's descent: The consequences of ecological instability. New York, William Morrow \& Co.

Quammen, D. (2012). Spillover: Animal infection and thenext human pandemic. New York, W.W. Norton \& Co. 
Rapoport, E. H. (1988). Lo bueno y lo malo tras el descubrimiento de América. El punto de vista ecológico y biogeográfico. Arbor 131 (513): 103-125.

Reyes-García, V, A. L. Balbo, E. Gómez-Baggethun, M. Gueze, A. Mesoudi, P. J. Richerson, X. RubioCampillo, I. Ruiz-Mallén y S. Shennan (2016). Multilevel processes and cultural adaptation: examples from past and present small-scale societies. Ecology and Society 21(4):2. https://doi.org/10.5751/ES-08561-210402

Richerson, P. J. y R. Boyd (2005). Not by genes alone: How culture transformed human evolution. Chicago: University of Chicago Press.

Rockström, J., D. Steffen, K. Noone, A. Persoon, F. S. Chapin III, E. Lambin, T. Lenton, M. Scheffer, C. Folke, J. Schelinhuber, B. Nykvist, C. A. de Wit, T. Hughes, S. van der Leeuw , H. Rodhe, S. Sörlin, P. K. Snyder, R. Costanza, U. Svedin, M. Falkenmark, L. Karlberg, R. W. Corell, V. J. Fabry, J. Hansen, B. Walker, D. Liverman, K. Richardson, P. Crutzen y J. Foley (2009). Planetary boundaries: Exploring the safe operating space for humanity. Ecology and Society 14(2): 32. http://www.ecologyandsociety.org/vol14/iss2/art32/

Roosevelt, A. C. (2013). The Amazon and the Anthropocene: 13,000 years of human influence in a tropical rainforest. Anthropocene 4: 69-87.

Rosenzweig, M. L. y R. D. McCord (1991). Incumbent replacement: evidence for long-term evolutionary progress Paleobiology 17: 202-213.

Ruddiman, W. F., E. C. Ellis, J. O. Kaplan y D. Q. Fuller (2015). Defining the epoch we live in. Science 348 (6230): 38-39. DOI: 10.1126/science.aaa7297

Sanderson, E., M. Jaiteh, M. Levy, K. Redford, A. Wannebo y G. Wolmer (2002). The human footprint and the last of the wild. Bioscience 52: 891-904.

Smith, B. D. (2007). Niche-construction and the behavioral context of plant and animal domestication. Evolutionary Anthropology 16: 188-199.

Smith, B. D. y M. A. Zeder (2013). The onset of the Anthropocene. Anthropocene 4: 8-13.

Steffen, W., P.J. Crutzen y J. R. McNeill (2007). The Anthropocene: are humans now overwhelming the great forces of Nature? AMBIO 36(8): 614-621.

Stringer, C. (2002). Modern human origins: progress and prospects. Philosophical Transactions of the Royal Society B 357: 563-579.

Stringer, C. (2016). The origin and evolution of Homo sapiens. Philosophical Transactions of the Royal Society B 371: 20150237. http://dx.doi.org/10.1098/rstb.2015.023

Subramanian, M. (2019). Anthropocene now: Influential panel votes to recognize Earth's new epoch. Nature. DOI:10.1038/d41586-019-01641-5

Svampa, M. y F. Longa (eds.) (2019). El Antropoceno como diagnóstico y paradigma. Lecturas desde América Latina. Utopía y Praxis Latinoamericana. Número Especial. 84. http://doi.org/10.5281/zenodo.2653159

Syvitski, J., C. N. Waters, J. Day, J. D. Milliman, C. Summerhayes, W. Steffen, J. Zalasiewicz, A. Cearreta, A. Gałuszka, I. Hajdas, M. J. Head, R. Leinfelder, J. R. McNeill, C. Poirier, N. L. Rose, W. Shotyk, M. Wagreich y M. Williams (2020). Extraordinary human energy consumption and resultant geological impacts beginning around 1950 CE initiated the proposed Anthropocene Epoch. Communications Earth \& Environment 1 (32). DOI:10.1038/s43247-020-00029-y 
Trischler, H. (2017). El Antropoceno, ¿un concepto geológico o cultural, o ambos? Desacatos: Revista de Antropología Social 54: 40-57.

United Nations (2019). Revision of world population prospects. https://population.un.org/wpp/

Vitousek, P. M., P. R. Ehrlich, A. H. Ehrlich y P. A. Matson (1986). Human appropriation of the products of photosynthesis. BioScience 36(6): 368-373.

Wackernagel, M., N. Schulz, D. Deumling, A. Callejas Linares, M. Jenkins, V. Kapos, C. Monfreda, J. Lohi, N. Myers, R. Norgaard y J. Randers (2002). Tracking the ecological overshoot of the human economy. PNAS 99: 9266-9271.

Wallerstein, I. (1979). The capitalist world economy. Cambridge, Cambridge University Press.

Wilson, D. S, y E. Sober (1994). Reintroducing group selection to the human behavioral sciences. Behavioral Brain Sciences 17:585-608. https://doi.org/10.1017/S0140525X00036104 
